\title{
Narrative review of pharmacotherapy for transthyretin cardiac amyloid
}

\author{
Jose B. Cruz Rodriguez ${ }^{1}$, Jose A. Tallaj ${ }^{2,3}$ \\ ${ }^{1}$ Division of Cardiovascular Diseases, Texas Tech University Health Science Center El Paso, El Paso, TX, USA; ${ }^{2}$ Division of Cardiovascular Diseases, \\ University of Alabama at Birmingham, Birmingham, AL, USA; ${ }^{3}$ Department of Medicine, Birmingham VA Medical Center, Birmingham, AL, USA \\ Contributions: (I) Conception and design: Both authors; (II) Administrative support: JB Cruz Rodriguez; (III) Provision of study materials or patients: \\ JB Cruz Rodriguez; (IV) Collection and assembly of data: Both authors; (V) Data analysis and interpretation: Both authors; (VI) Manuscript writing: \\ Both authors; (VII) Final approval of manuscript: Both authors. \\ Correspondence to: Jose B. Cruz Rodriguez, MD, MPH. Division of Cardiovascular Diseases, Texas Tech University Health Science Center El Paso, \\ 4800 Alberta Avenue, El Paso, TX 79905, USA. Email: Benjamin.cruz@ttuhsc.edu.
}

\begin{abstract}
Treatment of cardiac amyloidosis is determined by the amyloid type and degree of involvement. Two types of amyloid commonly infiltrate the heart: immunoglobulin light-chain amyloid (AL), and transthyretin amyloid (ATTR), that encompasses other two forms, a hereditary form (hATTR), and a sporadic, age-related wild-type (wtATTR). The prevalence is expected to increase with aging population. The natural history of ATTR cardiomyopathy includes progressive heart failure (HF), complicated by arrhythmias and conduction system disease. New therapies options have been approved or are under investigation. We performed a narrative literature review, manually-searched the reference lists of included articles and relevant reviews. Treatment for cardiac ATTR should be directed towards alleviation of HF symptoms and to slow or stop progressive amyloid deposition. Conventional HF medications are poorly tolerated and may not alter the disease progression or symptoms, except perhaps with the administration of diuretics. There are three approaches of therapy for ATTR cardiomyopathy: tetramer stabilizers, inhibition of ATTR protein synthesis and clearance of deposited fibrils. Tafamidis diminishes the progression of cardiomyopathy, functional parameters, improves overall outcome in patients with early disease stages, irrespective of ATTR status and is well tolerated. Diflunisal has shown promising results in early studies, but at the expense of significant side effects. Two new agents, antisense oligonucleotides, patisiran and inotersen are under investigation in cardiac amyloidosis. Patisiran appears to be the most effective treatment for hATTR, although evidence is limited, with a relatively small cardiac subpopulation. Therapies considering clearance of amyloid fibrils from tissue remain experimental. In conclusion, tafamidis is the only approved agent for the treatment of ATTR cardiomyopathy although multiple other agents have shown promising early results and are undergoing clinical trials. Careful consideration of the type of ATTR, comorbidities and disease stage will be key in deciding the optimal therapy for ATTR patients.
\end{abstract}

Keywords: Wild-type amyloid; hereditary amyloid; heart failure (HF); treatment

Submitted Jun 11, 2020. Accepted for publication Jul 30, 2020.

doi: 10.21037/atm-20-4636

View this article at: http://dx.doi.org/10.21037/atm-20-4636

\section{Introduction}

Systemic amyloidosis incorporates a family of diseases induced by misfolded proteins (1). The amyloid type is defined by the particular precursor protein that misfolds to form amyloid fibrils and dictates the patient's clinical course as well as treatment of cardiac amyloidosis. Consequently, early recognition and accurate classification are essential (2).

Amyloidosis is a group of diseases that affect multiple 
organs, particularly the central and peripheral nervous system, kidneys and heart. There are two types of amyloid that commonly infiltrate the heart: immunoglobulin lightchain (AL or primary systemic) amyloid, and transthyretin amyloid (ATTR). Other protein precursors that can infiltrate the myocardium such as apolipoprotein A have a very low prevalence $(<5 \%)$ and are not consider here. Cardiac amyloidosis may occur in patients with Amyloid A (AA) amyloidosis due to chronic inflammatory diseases (3). Transthyretin-related amyloidosis (ATTR) includes two forms-familial disease arising from misfolding of a mutated transthyretin (familial, variant or hereditary amyloid cardiomyopathy hATTR, and a sporadic, non-genetic disease due to misaggregation of wild-type transthyretin (wtATTR), also known as senile systemic amyloidosis (1).

AL amyloidosis has an approximate incidence of 2,500 new cases annually (4) with cardiac involvement in approximately $50 \%$ of cases $(5,6)$. The prognosis with $\mathrm{AL}$ disease is poor, with median survival of $<1$ year in the presence of heart failure (HF) symptoms (6). Cardiac involvement is a major determinant of poor outcome, even after bone marrow transplantation. Unlike AL amyloidosis, ATTR cardiomyopathy is slowly progressive and better tolerated clinically.

Transthyretin, formerly misnamed prealbumin, is a 127 -amino acid, $56 \mathrm{kDa}$ transport protein primarily secreted by the liver, with lesser amounts produced by the choroid plexus for cerebral spinal fluid and retinal pigmented epithelial cells for the vitreous of the eye $(1,7)$. Normally, transthyretin circulates as a homotetramer but due to genetic mutation (hATTR) or aging (wtATTR), tetramers can dissociate to monomers that misassemble into amyloid fibrils $(8,9)$.

There are emerging therapies for ATTR cardiomyopathy. For this analysis, we performed a narrative literature review on the topic aiming to clarify the current role of the potential pharmacologic therapies for ATTR. We searched the Cochrane Central Register of Controlled Trials (CENTRAL), Cumulative Index to Nursing and Allied Health Literature (CINAHL), Embase, and PubMed databases through March 31st, 2020, with no restrictions on language. Key words of cardiac amyloidosis, amyloid cardiomyopathy treatment and tafamidis were utilized. Large prospective studies and systematic reviews were included, with notorious lack of metanalysis in the topic. We manually-searched the reference lists of included articles and relevant reviews.

We present the following article in accordance with the
NARRATIVE REVIEW reporting checklist (available at http://dx.doi.org/10.21037/atm-20-4636).

\section{Wild type ATTR}

In wtATTR transthyretin becomes unstable and has an increased tendency to accumulate and form fibrils without genetic abnormalities (9). By unclear aging mechanisms, accumulation and cardiac deposition of genetically normal ATTR increase over time $(1,9)$, suggesting that we can expect the incidence of wtATTR to increase with the aging population. It has been suggested that cardiac wtATTR could explain up to $15 \%$ of cases of HF in older adults (7).

Prevalence of wtATTR increases with age and practically all patients are over 60 years of age at diagnosis (1). This variant is a gender-specific disease, exhibiting approximately 25-50:1 male:female ratio (10). Patients usually manifest isolated, strong cardiac involvement, which may be preceded by carpal tunnel syndrome by an average of 5 to 10 years $(11,12)$. Autopsy studies have identified cardiac wtATTR deposition in over $25 \%$ of individuals above 80 years of age (13). Other autopsy studies have shown that $17 \%$ of patients with $\mathrm{HF}$ with preserved ejection fraction (EF) and even $5 \%$ of healthy controls had wtTTR amyloid depositions (14).

\section{Variant ATTR}

Variant ATTR (hATTR) has an autosomal dominant trait with variable penetrance and it is also known as familial amyloid cardiomyopathy. The gene is located on chromosome 18q12.1, with over 130 missense pathogenic mutations identified $(15,16)$. These mutations are clustered into geographic and/or ethnic groups (1). In general, hATTR is heterogeneous based on age, with neuropathy phenotype predominating in early-onset cases and cardiac dysfunction phenotype predominating in later-onset cases, similar to wtATTR (9).

The most common mutation in the United States of America (USA) is Val122Ile (7) and is present in up to $3-4 \%$ African Americans older than 65 years of age, which represents 1.5 million subjects being allele carriers in the US (17). This implies that the Val122Ile mutation a potentially important cause of HF in the elderly Black community (18). Fortunately, the penetration is variable and low, especially in female carriers. The second most common mutation in the US is Thr60Ala, which originates in the Northern part of Ireland and causes a mixed phenotype with 
high prevalence of carpal tunnel syndrome (19). Worldwide, Val30Met (pV50M) is the most common mutation with late-onset presentation, predominantly hATTR polyneuropathy phenotype and endemic in certain regions of Portugal, Japan, and Sweden (7). Regardless of patient age, transthyretin genetic testing should be performed in all patients with ATTR as it can have implications for family members at risk, although there are no clear guidelines on how to perform surveillance of disease in carriers.

Increasingly data support the fundamental role of circulating or pre-fibrillar amyloidogenic proteins for cardiac dysfunction in AL, wtATTR and hATTR $(1,20)$. Data generated from both $\mathrm{AL}$ and ATTR models of disease evidence that pre-fibrillar protein aggregates are the ones that contribute to organ toxicity, not mature amyloid fibrils (1).

\section{Diagnosis}

The diagnosis of cardiac amyloidosis has had a rather significant shift in the last $5-10$ years. There is major emphasis now to diagnose the disease non-invasively. A contemporary approach to non-invasive diagnosis of cardiac ATTR includes echocardiography with strain imaging, cardiac magnetic resonance (CMR), electrocardiography (ECG), nuclear scintigraphy, and serum biomarker testing (1). We present some diagnostic pearls as multiple diagnostic algorithms have been proposed $(21,22)$ and will not be discussed in detail in this review. Our diagnostic approach is presented in Figure 1.

Low-voltage criteria by electrocardiogram is noted in only $25 \%$ to $40 \%$ of patients with ATTR (23). In fact, in patients with Val122Ile, 25\% met criteria for left ventricular (LV) hypertrophy (24).

Although similar findings have been reported in ATTR cardiomyopathy, most echocardiographic features are derived from the AL population (10). Typical characteristics include thick ventricular wall with "speckling" appearance of the myocardium, small LV chamber volume, valvular thickening, atrial enlargement and signs of elevated filling pressures due to restrictive diastolic filling. Interestingly, septal wall hypertrophy can occur asymmetrically in up to a quarter of patients with wtATTR, confounding the diagnosis of ATTR with hypertrophic cardiomyopathy (25).

Another useful tool for the identification of cardiac involvement in $\mathrm{AL}$ disease is longitudinal strain measurement by tissue Doppler and echo speckle tracking (26). Typical findings of cardiac amyloidosis include decrease in longitudinal function and regional longitudinal strain pattern with predominant reduction of strain in basal and mid-ventricular segments along with normal strain in apical segments ("apical sparing") (27).

In the absence of a monoclonal protein suggestive of AL, nuclear scintigraphy could be used to make the diagnosis of cardiac ATTR noninvasively with Tc-99mpyrophosphate (PYP) and Tc-99m 3,3-diphosphono1,2-propanodicarboxylic acid (DPD) or Tc-99mhydroxymethylene diphosphonate (HMDP). Only PYP is available in the US (21). Nuclear scintigraphy should always be performed concomitant or after screening for monoclonal protein in the diagnostic evaluation. If the nuclear scan is positive, a genetic test may differentiate between hATTR and wtTTR.

On CMR, the presence of diffuse subendocardial or transmural enhancement patterns or failure to suppress the myocardial signal suggests amyloidosis (28).

Endomyocardial biopsy continues to be the gold standard for ATTR cardiomyopathy diagnosis and is virtually $100 \%$ sensitive and specific if $\geq 4$ different biopsy specimens are collected and evaluated for amyloid deposits by Congo red staining (29). Immunohistochemistry or mass spectrometry can determine the precursor protein. Fat aspirate, a common diagnostic test, has a very poor sensitivity in ATTR disease, reported around 15\% (30). Our approach has always been to biopsy the organ that we know it is affected, in this case, the heart.

\section{Clinical course and prognosis}

The natural history of ATTR cardiomyopathy is more indolent than AL amyloid, and includes progressive HF symptoms, accompanied by atrial arrhythmias and conduction system disease. Peripheral and autonomic neuropathy can occur in wtATTR, but it is usually less severe than that in hATTR and the overall prognosis seems to be determined by the degree of cardiac involvement. In both types of ATTR, depressed left ventricular ejection fraction (LVEF), defined as $<50 \%$ is associated with reduced survival (27).

Cardiac wtATTR is almost exclusively a disease of older males with predominant cardiac involvement (21). It has been proposed that $13 \%$ of patients hospitalized for HF with preserved $\mathrm{EF}$ and $16 \%$ of patients with severe aortic stenosis undergoing valve replacement had ATTR amyloid, with a large percentage of them meeting criteria for lowflow, low gradient aortic stenosis (7). Transthyretin disease has better prognosis and survival than AL amyloidosis, but 


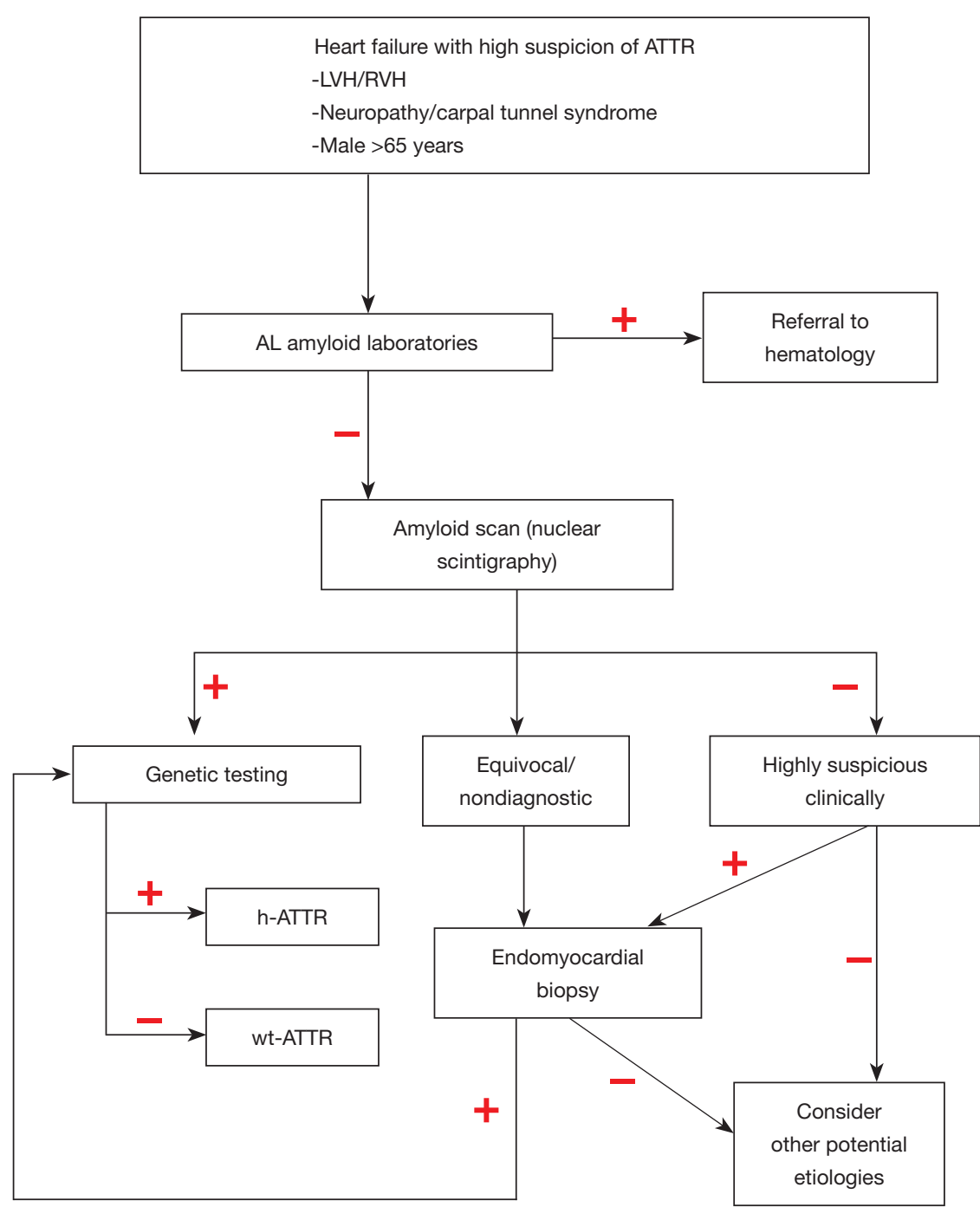

Figure 1 Diagnostic algorithm for ATTR. ATTR, transthyretin amyloid; h-ATTR, hereditary ATTR; LVH, left ventricular hypertrophy; RVH, right ventricular hypertrophy; wtATTR, wild-type ATTR.

tends to evolve to intractable HF and death due to systolic HF or dysrhythmia.

The prospective, multicenter and observational Transthyretin Amyloidosis Cardiac Study (TRACS) (31) comparing V122I hATTR to wtATTR found that survival was lower for V122I patients (26 compared to 46 months, respectively). It is important to note that these survival estimates could be biased by the advanced cardiac amyloidosis involvement at the time of referral. Survival in wtTTR disease appears most favorable of the amyloid cardiomyopathies, particularly compared to Val122Ile (31). Reported median survival for wtTTR varies in the range of $43-75$ months, with a much slower progression $(10,32,33)$.
The Mayo Clinic wtATTR-CM staging system (34) used thresholds of biomarkers, troponin $\mathrm{T}>0.05 \mathrm{ng} / \mathrm{mL}$ and $\mathrm{N}$-terminal pro-B-type natriuretic peptide $>3,000 \mathrm{pg} / \mathrm{mL}$ to classify in three stages where stage I has both biomarker values below threshold; stage II: 1 biomarker above the threshold; and stage III: both above the threshold. This staging system translates to median survival of 66,42 , and 20 months, respectively.

Echocardiographic findings including LV mass, wall thickness and diastolic function are not independent predictors of survival. Typically associated with a preserved $\mathrm{EF}$, cardiac amyloidosis can present with reduced EF (defined as $<40 \%$ ) in $30 \%$ to $50 \%$ of wtATTR patients 
$(27,34)$. Conduction abnormalities are more common in wtATTR than hATTR cardiomyopathy, with up to $33 \%$ of patients requiring permanent pacemakers, which can occur years before onset of HF (27). Atrial arrhythmias are also more prevalent in wtATTR cardiomyopathy than in hATTR, occurring in $40 \%$ to $60 \%$ of patients at diagnosis in recent series $(27,34)$. Ventricular arrhythmias do not seem to be significantly prevalent in this population.

\section{Treatment}

The optimal treatment approach in cardiac ATTR should be directed towards alleviation of HF symptoms, and at the same time to slow or stop progressive amyloid deposition (1).

ATTR cardiomyopathy progresses with bi-ventricular thickening, diastolic dysfunction from loss of compliance and congestive HF symptoms from elevation in cardiac filling pressures (1). Fluid status optimization is the fundamental aspect in the management of cardiac amyloidosis, mostly accomplished by administration of loop diuretics and spironolactone. Frequently, acute or chronic renal dysfunction complicate management of $\mathrm{HF}$, and over diuresis precipitates hypotension. Progressive cardiorenal disease is an important marker of worsening disease and poor outcome (35). In our experience, once the renal function starts deteriorating, it is the time of rapid decline.

As ATTR progresses, impairing diastolic filling and reducing the stroke volume that is fixed, tachycardia is the only compensatory mechanism that maintains cardiac output (36). Hence, beta-blockers are frequently poorly tolerated as they inhibit compensatory tachycardia and have a negative inotropic effect in amyloid affected hearts (1). The use of low-dose beta-blockers might be helpful only if tachyarrhythmia is present (21). It has been suggested that the intolerance of beta-blockers in newly diagnosed HF should increase the diagnostic suspicion of amyloidosis (7).

Calcium channel blockers $(37,38)$ and digitalis (39) are contra-indicated in cardiac amyloidosis as they bind to amyloid fibrils and have been reported to increase drug toxicity. Other typical agents for HF, like angiotensin receptor blockers, angiotensin converting enzyme inhibitors or neprilysin inhibitors have not been associated with improved outcomes or symptoms (1). In fact, it may lead to additional hypotension, which is common in patients with ATTR amyloid.

Rhythm control with successful maintenance of sinus rhythm in atrial fibrillation is challenging and frequently unsuccessful in the long term as the increased ventricular end-diastolic pressures promote atrial dilation, as well as the atrial amyloid fibrils infiltration (1). However, due to reduced atrial mechanics, the atrial contribution to ventricular filling is minimal or absent. In fact, these patients tend to do better in atrial fibrillation with ventricular rates in the low $100 \mathrm{~s}$, than sinus rhythm in the 60-80 bpm range. Even when rhythm control is accomplished, cardiac amyloid infiltration, especially of the AL type, is associated with a high incidence of cardioembolic events. Grade II or III diastolic dysfunction, dilated atriums, and reduction in mitral A wave less than $19-23 \mathrm{~cm} / \mathrm{s}$ are risk factors for intracardiac thrombus formation. Interestingly, in some series, $20 \%$ of patients without documented atrial fibrillation have intracardiac thrombi $(40,41)$. Thus, anticoagulation is recommended if the E-wave mitral inflow velocity $\leq 20 \mathrm{~cm} / \mathrm{s}$ using spectral Doppler and no other contraindications are present (41). Furthermore, regardless of the CHADS-VASc score, long-term anticoagulation is recommended once atrial fibrillation is detected in ATTR (7).

Amiodarone increases the risk of heart block in cardiac ATTR, although patients must also be monitored for the known toxicities (42). Stablished guidelines should be followed to consider placement for permanent pacemaker. High index of suspicious, with early Holter monitoring should be consider with any patient with symptoms of syncope or palpitations. It is important to recognize that these patients will likely have increased rates of right ventricular pacing which could lead from LV dyssynchrony (9). Nonetheless, there is no data to suggest that these patients benefit from biventricular pacing and regular guidelines should be follow for cardiac resynchronization therapy.

Ventricular arrhythmias do not seem to be prevalent in this population. The principal cause of arrhythmic death in high-risk AL patients was pulseless electrical activity in our experience as well as a case series (43). Likely because of this, implantable cardioverter-defibrillator (ICD) implantation did not improve overall survival for primary prevention $(44,45)$. Secondary prevention implant is reasonable per current guidelines, but the decision should be individualized.

Patients with amyloidosis often develop gastrointestinal disturbance and is critical to optimize nutritional status to contribute to fluid balance management via improvement of oncotic pressure. Nausea and early satiety can be managed with domperidone. Orthostatic hypotension, likely a combination of low output, small stroke volume and autonomic dysfunction, can be treated with midodrine or pyridostigmine (9). 


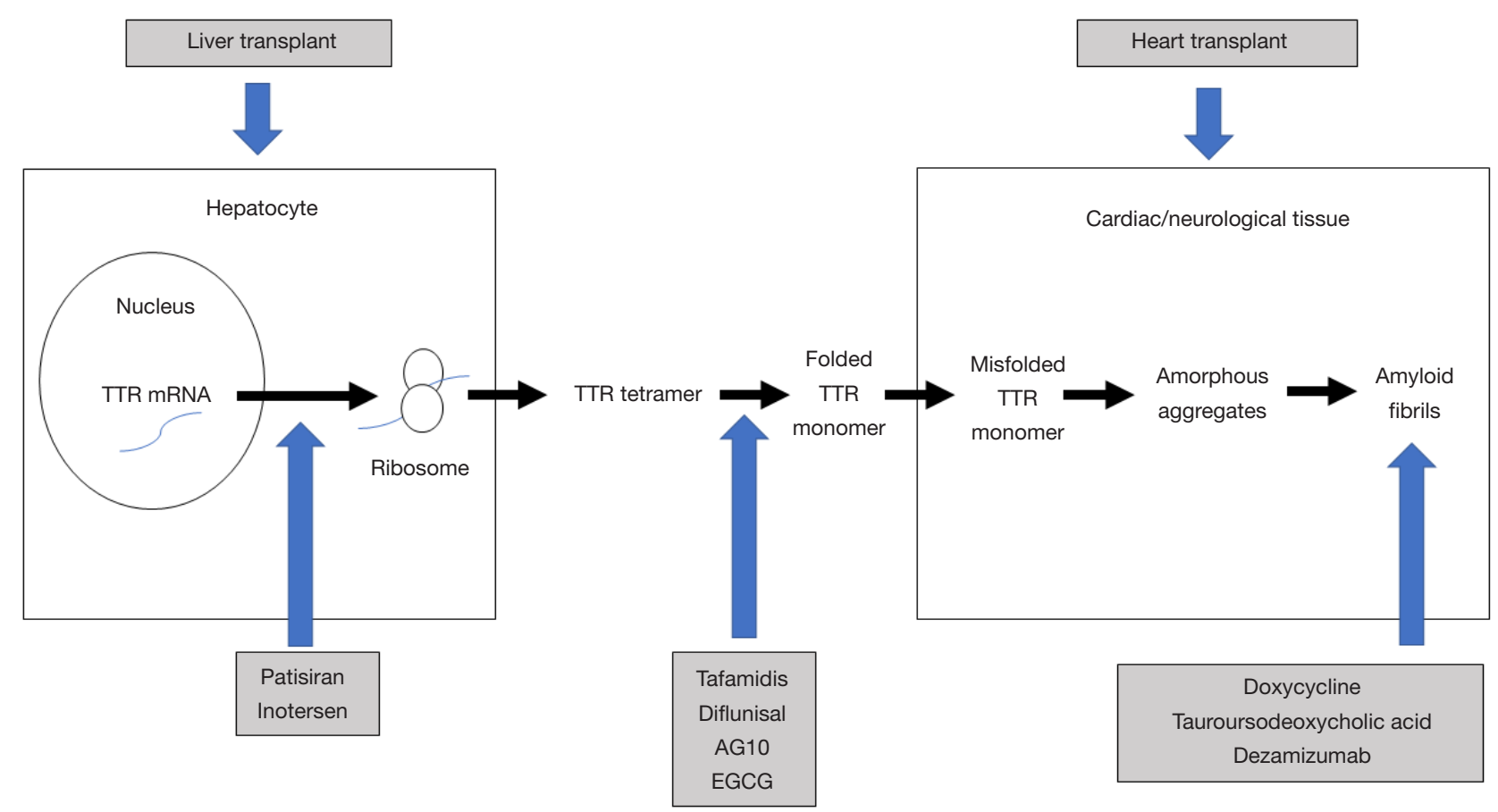

Figure 2 Therapeutic strategies for ATTR. EGCG, epigallocatechin-3-gallate; mRNA, messenger ribonucleic acid; TTR, transthyretin; ATTR, transthyretin amyloid.

\section{Amyloid-specific therapies}

Figure 2 presents the mechanism of action of the therapeutic strategies for ATTR, that we discuss in the following sections.

\section{Tetramers stabilizers (molecule inhibitors)}

Tafamidis is a benzoxazole derivative that covalently binds to thyroxine binding sites on transthyretin tetramers. The binding inhibits dissociation into monomers and inhibits aggregate formation both in vitro and in vivo $(9,46)$. The transthyretin stabilization has been reported from $89 \%$ to $100 \%$ at various time points in different trials $(47,48)$. Tafamidis is approved in Europe for stage I polyneuropathy (oral, $20 \mathrm{mg}$ once daily) and the USA for cardiomyopathy (oral, $80 \mathrm{mg}$ tafamidis meglumine or $61 \mathrm{mg}$ tafamidis daily) in adults with both hATTR and wtATTR (11).

Peak plasma concentration is achieved at a median time of 2 hours, and can be delayed by simultaneous intake of food, although food does not change the absorption. Steady state is reached after 14 days with continuous administration of tafamidis $20 \mathrm{mg}$ once daily (11). Tafamidis is predominantly protein-bound in $99.9 \%$ and metabolized and excreted by glucuronidation in the liver with a slow half-life of 59 hours (49).

After 30 months of follow-up in patients with wtATTR and hATTR $(\mathrm{n}=441)$, the randomized, placebo-controlled ATTR-ACT trial (50) showed that tafamidis significant reduced cardiovascular-related hospitalizations by a third and all-cause mortality by $30 \%$ and $(20$ and $80 \mathrm{mg}$ once daily pooled, $\mathrm{n}=264$ ). After 2.5 years of treatment, this translates to a number needed to treat of 7.5 to prevent one death. The mortality benefit was superior in patients with early-stage cardiac disease (NYHA class I or II) and seen in both transthyretin subtypes. Although in-vitro results suggested that higher doses are better, in ATTR-ACT the difference between the 20 and $80 \mathrm{mg}$ dose was not clinically significant. Functional capacity, assessed via the 6-minute walk test and quality of life declined significantly slower in patients in the tafamidis arm. Differences in survival only reached significance after treatment duration of 18 months, although slow deterioration of walking distance and quality of life were seen as early as 6 months.

The findings in ATTR-ACT support that tafamidis is most efficacious if administered early in the course of the disease, likely because tafamidis acts by stabilizing tetramers instead of eliminating fibrils (51). In fact, in the ATTR-ACT patients with NYHA class III at baseline had 
higher rates of cardiovascular hospitalizations compared to placebo. Tafamidis is considered well-tolerated and despite some reports of higher urinary tract infection or effect in QTc interval in polyneuropathy patients, that effect has not been seen in cardiac patients $(11,52)$. The results of ATTR-ACT corroborate that tafamidis is better in halting the progression of cardiomyopathy in ATTR patients when compared to placebo, particularly if the earlier course of the disease.

Diflunisal is a nonsteroidal anti-inflammatory drug with transthyretin-stabilizing effects in both vATTR and vATTR. Although it is not specifically approved for ATTR treatment in any country, diflunisal can be used off-label (11) for ATTR associated cardiomyopathy. The recommended dose is $250 \mathrm{mg}$ twice daily, which is lower than the dose for anti-inflammatory effects, resulting in inhibition of fibrils formation and deposition $(43,53)$. In a small singlearm, open-label study, diflunisal use was associated with no significant changes in LV mass index and EF, suggesting that it could stabilize cardiac function at 12 months (54). In another small and nonrandomized study, diflunisal had a survival benefit similar to tafamidis (55). Given that a generic formulation is available, it could be considered as a treatment option in countries where other treatments would be financially prohibitive. As with other nonsteroidal anti-inflammatories, the chronic use of diflunisal is associated with nephrotoxicity, thrombocytopenia, fluid retention, gastrointestinal and cardiac side effects have limited the use of diflunisal in patients with ATTR $(10,21)$. It is recommended to provide prophylaxis for peptic ulcer disease, monitor of platelet counts and close renal monitoring including volume status to minimize the risk for side effects (11). Tafamidis attaches to the transthyretin tetramer more tightly, but diflunisal needs to achieve high serum to bind with transthyretin with weaker binding coefficients. Although promising, diflunisal has not been proven to be beneficial in randomized studies.

AG10 is another transthyretin stabilizer currently being investigated. In a small phase II trial $(n=49$, with both wtATTR and hATTR), AG10 showed consistent stabilization of transthyretin and restored serum ATTR concentrations to normal clinical range (56). It was well tolerated and safe in 28 days in NYHA class II and III subjects. A phase III study (57) is been ongoing (Study of AG10 in Amyloid Cardiomyopathy, NCT03458130).

The FDA approved Parkinson's disease medication, catechol-O-methyltransferase inhibitor tolcapone, also has transthyretin protein stabilization properties and is presently under investigation (58).

Epigallocatechin-3-gallate (EGCG), the primary catechin in green tea, stabilizes serum transthyretin tetramers by binding to a different site than tafamidis and diflunisal (59), avoiding concerns that it may compete with other tetramer stabilizers. It inhibits pre-fibrillary transthyretin deposition and disrupts mature amyloid transthyretin fibrils in hATTR transgenic mice $(60,61)$. Furthermore, EGCG potentially can disrupt preformed fibrils and clear amyloid deposits by converting them into unstructured off-pathway oligomers (62). Although synergistic effect with tafamidis or diflunisal in stabilizing transthyretin has been reported in-vitro (63), that has not been demonstrated in-vivo and given the interaction of EGCG with serum albumin, the bioavailability and transport of tafamidis could be compromised by EGCG use (64). Small observational reports ( $\mathrm{n}=14,53 \%$ hATTR and 47\% wtATTR) showed that EGCG $550 \mathrm{mg}$ daily for 12 months significantly reduced interventricular septal thickness and LV myocardial mass (65), presumedly due to a decrease of amyloid fibrils load in the tissue (66).

\section{Inhibition of TTR mRNA and protein synthesis}

Antisense oligonucleotides (ASOs) work as antisense strands to specific mRNA sequences coding for pathogenic proteins (like wtATTR and vATTR) and control the expression of that mRNA (67). After binding to the garget mRNA, ASO control the transcription by steric blocking (occupancy) or enzymatic mechanisms inducing RNA cleavage or degradation (68). Both ASOs manifest cardiac effects after 15-18 months of therapy, much later than neurologic improvement, that was observed at 8-9 months. Both are approved for use in patients with neurological involvement, but not for cardiac involvement. However, their use may be limited given the potential side effects and the costs associated with these agents (69).

Patisiran is a double stranded RNA oligonucleotide that enters the hepatocyte cytoplasm by lipid nanoparticle. Its RNA double strand binds to Ago2 in the RNA-induced silencing complex interfering by releasing the inactive sense strand. Ago2 works as an endonuclease that splits RNA in RNA-RNA duplexes $(11,70)$, leading to degradation and lowering transthyretin protein levels up to $90 \%$ (71-73). Patisiran is administered every 3 weeks intravenously. It is recommended to premedicate with dexamethasone, oral acetaminophen, an H2-blocker and an H1-blocker due to the risk of infusion-related reactions, that can happen in up to $20 \%$ of patients (74). The APOLLO-II 
trial $(n=225)$ reported at 18 months mean reductions in serum transthyretin of $81 \%$, regardless of the mutation, which translated to improvements in neuropathy (75). In a subgroup analysis of the APOLLO trial (76), 56\% of the study participants had evidence of cardiac involvement $(\mathrm{n}=126)$ and patisiran showed improvement in $\mathrm{LV}$ wall thickness, $\mathrm{N}$-terminal pro-brain natriuretic peptide compared to both baseline and placebo. There was also improvement in global longitudinal strain pattern, particularly at the base and increase in cardiac output (76) in patients in the early course of the disease, since patients with NYHA class III or IV were excluded. A reduction in all-cause hospitalization $(-50 \%)$ and all-cause mortality $(-45 \%)$ was also seen $(75,76)$. Overall, patisiran prevents the worsening of myocardial dysfunction in most patients, but there is minimal improvement. Transthyretin works as a transporter for retinol-binding protein, so reductions in serum transthyretin can lead to reduction in serum vitamin A (71) and supplementation with vitamin A is recommended, at least 3,000 IU daily (11). The safety profile of patisiran is extrapolated from small studies, which raises concern as the phase III trial of another ASO with same mechanism of action, revusiran, had to be stopped prematurely due to increase mortality in the treatment arm (77). Studies with larger number of participants are warranted to further define the safety profile of patisiran.

Inotersen is a single stranded ASO that specifically suppresses transthyretin RNA reducing serum concentrations up to $80 \%$. It is chemically modified to improve binding affinity, provide stability against enzymatic cleavage, and reduce pro-inflammatory products (68). Inotersen attaches to hATTR or wtATTR mRNA forming DNA-RNA duplexes, that activate enzymatic degradation $(11,78)$. It has only been studied for hATTR with polyneuropathy and despite reducing peripheral neuropathy, the benefits were offset by glomerulonephritis (3\%) and thrombocytopenia (3\%) (79). In a small study $(\mathrm{n}=22)$, at 12 -month follow-up it showed stabilization in LV thickness and dimensions (80), but other secondary analysis have not shown difference in echocardiographic parameters compared to placebo (79). In subset of the NEURO-TTR randomized study, $62 \%$ of patients had cardiac involvement and after a median follow-up of 480 days, the risk of side effects did not increase with prolonged exposure to inotersen (81). Similar to patisiran, vitamin A supplementation is recommended (79). It is administered subcutaneously once a week and the half-life is approximately 32 days (82). If used, it is recommended to monitor platelets and renal function including proteinuria every 2-3 weeks (11).

\section{Clearance of amyloid fibrils}

Currently no available treatment to remove amyloid fibrils already deposited in cardiac or neurological tissue (21). The antibiotic doxycycline has been shown to inhibit amyloid fibril formation, disrupting accumulated mature fibrils (83). The combination of doxycycline and tauroursodeoxycholic acid demonstrated strong synergistic effect in the clearance and reabsorption of ATTR fibrils (84). This combination improved global longitudinal strain by $5 \%$ in one-third of patients (85). Those with improvement were younger, had lower biomarker levels and NYHA stage at baseline, suggestive earlier courses of the disease. Nonetheless, NYHA functional class, cardiac biomarkers and EF did not improve with the combination therapy. The halting effect in disease progression is promising, as almost half of patients in that cohort had at baseline NYHA class III or IV (85). A phase II study $(n=20)$ on patients with both hATTR and wtATTR with daily administration of oral doxycycline $(100 \mathrm{mg})$ and tauroursodeoxycholic acid (250 $\mathrm{mg}$ three times a day), reported unchanged neuropathy symptoms with no clinical progression of cardiac involvement at 12 months (86). These agents are used in patients with AL amyloid as well, although there is limited clinical data (11).

Other prospective therapeutic option is antibodymediated (anti-transthyretin) removal of amyloid aggregates by targeting transthyretin residues. During the formation of pathological amyloid fibrils, the conformational variations uncover epitopes on the molecular surface of ATTR, internalized in the tetramer but exposed in the monomer (87), and these epitopes serve as the antibody target (88). Binding of the antibody prevents transthyretin fibril formation in a dose-dependent fashion, inhibiting the formation of new amyloid deposits, and promoting macrophage phagocytosis of aggregated fibrils, which leads to reduce fibrils in previously affected tissues $(89,90)$. Although promising, the human studies have resulted in cessation due to toxicity or futility.

Dezamizumab is an IgG antibody that targets the nonfibrillar plasma glycoprotein serum amyloid P (SAP), present in all subtypes of amyloid (91). Dezamizumab specifically reduces amyloid deposits by targeting SAP and activating complement pathways as well as macrophage-dependent phagocytosis (92). In a phase I trial, dezamizumab reduced amyloid content in kidney and liver 
in a dose-dependent manner (93). Transient changes in NT-proBNP were observed, although no effect on cardiac amyloid deposits were shown (93).

\section{Transplantation}

Because of potential rapid deterioration, early referral to a transplantation center is recommended in patients with amyloid cardiomyopathy. Moreover, the success of mechanical support given the small LV chamber and significant left ventricular hypertrophy (LVH) is unlikely. Mutant ATTR protein is produced in the liver so for hATTR, combined orthotopic heart and liver transplantation (OHT and OLT, respectively) could be considered. If only OHT is performed, there have been reports of recurrence in the transplanted heart (9), particularly non-V30M ATTR (94). Survival post-transplant is excellent, when compared with patients with $\mathrm{AL}$ amyloid. Five-year survival post-OHT is $38 \%$ in AL patients, $67 \%$ at 2-year follow-up in wtATTR patients and $100 \%$ at 3 years in hATTR recipients $(95,96)$. Notoriously, current guidelines do not recommend OHT in patients older than 70 years of age which precludes many patients with wtATTR $(9,97)$. An approach that is getting a lot of traction is to perform OHT alone in patients with hATTR, followed by life-long treatment with tafamidis.

\section{Future directions}

With the exception of tafamidis, we lack studies analyzing the effects of different medications with ATTR-associated cardiomyopathy as primary endpoints. Moreover, the need of "hard outcomes" as survival or cardiac events should be the primary outcome, instead of surrogate endpoints as biomarkers or echocardiographic parameters. It remains questionable whether every amyloid deposit has pathologic significance and requires treatment, as it has been documented a high burden of amyloid deposit in autopsy studies $(13,14)$. Like minimal vascular calcifications, wtTTR amyloid deposits may be a physiologic sign of aging to a certain extent (11). Noninvasive risk stratification strategies will have to develop to identify those in need of treatment. Given its low penetrance, it is uncertain if treatment for carriers of genetic mutations known to cause ATTR will reduce the disease progression, and at which point the treatment needs to be initiated. Other strategies remain "off-label", though we have endorsed in our practice, like offering asymptomatic carriers diflunisal when they reach an age at which disease is likely to penetrate or if they demonstrate tissue amyloid infiltration confirmed by biopsy or bone scintigraphy. Clinical trials exploring the combinations of inhibition of tetramer stabilizers, ASOs and removing existing amyloid deposits would theoretically pose the biggest benefit, particularly in those with advance disease.

\section{Summary}

Tafamidis is the only approved agent for the treatment of ATTR cardiomyopathy, although multiple other agents are undergoing clinical trials. Clinicians need to be aware and vigilant on a daily basis to suspect ATTR cardiomyopathy in the right clinical scenario, particularly with the advances presented in this article for the noninvasive diagnosis. The demonstration of efficacy and the anticipated regulatory approval of more therapies. Future clinical practice will have to promote individualized decision-making to select the best agent depending on the type of transthyretin, mutations, disease stage and comorbidities (11).

\section{Acknowledgments}

Funding: None.

\section{Footnote}

Provenance and Peer Review: This article was commissioned by the Guest Editors (Dr. Debabrata Mukherjee and Dr. Jose B. Cruz Rodriguez) for the series "Heart Failure Update and Advances in 2021" published in Annals of Translational Medicine. The article was sent for external peer review organized by the Guest Editors and the editorial office.

Reporting Checklist: The authors have completed the NARRATIVE REVIEW reporting checklist. Available at http://dx.doi.org/10.21037/atm-20-4636

Conflicts of Interest: Both authors have completed the ICMJE uniform disclosure form (available at http://dx.doi. org/10.21037/atm-20-4636). The series "Heart Failure Update and Advances in 2021" was commissioned by the editorial office without any funding or sponsorship. JBCR served as the unpaid Guest Editor of the series. JAT has no other conflicts of interest to declare.

Ethical Statement: The authors are accountable for all 
aspects of the work in ensuring that questions related to the accuracy or integrity of any part of the work are appropriately investigated and resolved.

Open Access Statement: This is an Open Access article distributed in accordance with the Creative Commons Attribution-NonCommercial-NoDerivs 4.0 International License (CC BY-NC-ND 4.0), which permits the noncommercial replication and distribution of the article with the strict proviso that no changes or edits are made and the original work is properly cited (including links to both the formal publication through the relevant DOI and the license). See: https://creativecommons.org/licenses/by-nc-nd/4.0/.

\section{References}

1. Ruberg FL, Berk JL. Transthyretin (TTR) cardiac amyloidosis. Circulation 2012;126:1286-300.

2. Dubrey SW, Hawkins PN, Falk RH. Amyloid diseases of the heart: Assessment, diagnosis, and referral. Heart 2011;97:75-84.

3. Gertz MA, Benson MD, Dyck PJ. Diagnosis, prognosis, and therapy of transthyretin amyloidosis. J Am Coll Cardiol 2015;66:2451-66.

4. Kyle RA, Linos A, Beard CM, et al. Incidence and natural history of primary systemic amyloidosis in Olmsted County, Minnesota, 1950 through 1989. Blood 1992;79:1817-22.

5. Madan S, Kumar SK, Dispenzieri A, et al. Highdose melphalan and 30 peripheral blood stem cell transplantation for light-chain amyloidosis with cardiac involvement. Blood 2012;119:1117-22.

6. Dubrey SW, Cha K, Anderson J, et al. The clinical features of immunoglobulin light-chain (AL) amyloidosis with heart involvement. QJM 1998;91:141-57.

7. Ruberg FL, Grogan M, Hanna M, et al. Transthyretin amyloid cardiomyopathy: JACC state-of-the-art review. J Am Coll Cardiol 2019;73:2872-91.

8. Kelly JW. Mechanisms of amyloidogenesis. Nat Struct Biol. 2000;7:824-6.

9. Bart NK, Thomas L, Korczyk D, et al. Amyloid Cardiomyopathy. Heart Lung Circ 2020;29:575-83.

10. Ng B, Connors LH, Davidoff R, et al. Senile systemic amyloidosis presenting with heart failure: A comparison with light chain-associated amyloidosis. Arch Intern Med 2005;165:1425-9.

11. Müller ML, Butler J, Heidecker B. Emerging therapies in transthyretin amyloidosis - a new wave of hope after years of stagnancy? Eur J Heart Fail 2020;22:39-53.

12. Nakagawa M, Sekijima Y, Yazaki M, et al. Carpal tunnel syndrome: a common initial symptom of systemic wild-type ATTR (WtATTR ) amyloidosis. Amyloid 2016;23:58-63.

13. Tanskanen M, Peuralinna T, Polvikoski T, et al. Senile systemic amyloidosis affects $25 \%$ of the very aged and associates with genetic variation in alpha2-macroglobulin and tau: a population-based autopsy study. Ann Med 2008;40:232-9.

14. Mohammed SF, Mirzoyev SA, Edwards WD, et al. Left ventricular amyloid deposition in patients with heart failure and preserved ejection fraction. JACC Heart Fail 2014;2:113-22.

15. Benson MD, Kincaid JC. The molecular biology and clinical features of amyloid neuropathy. Muscle Nerve 2007;36:411-23.

16. Finsterer J, Iglseder S, Wanschitz J, et al. Hereditary transthyretin-related amyloidosis. Acta Neurol Scand 2019;139:92-105.

17. Buxbaum JN, Ruberg FL. Transthyretin V122I (pV142I)* cardiac amyloidosis: an age-dependent autosomal dominant cardiomyopathy too common to be overlooked as a cause of significant heart disease in elderly African Americans. Genet Med 2017;19:733-42.

18. Rastogi S, Johnson T, Hoeffel E, et al. The black population: 2010. 2010 Census Briefs. Accessed April 6th 2020. Available online: https://www.census.gov/prod/ cen2010/briefs/c2010br-06.pdf

19. Reilly MM, Staunton H, Harding AE. Familial amyloid polyneuropathy (TTR ala 60) in north west Ireland: a clinical, genetic, and epidemiological study. J Neurol Neurosurg Psychiatry 1995;59:45-9.

20. Buxbaum JN, Tagoe C, Gallo G, et al. Why are some amyloidoses systemic? Does hepatic "chaperoning at a distance" prevent cardiac deposition in a transgenic model of human senile systemic (transthyretin) amyloidosis? FASEB J 2012;26:2283-93.

21. Kristen AV. Amyloid cardiomyopathy. Herz 2020;45;267-71.

22. Maurer MS, Bokhari S, Damy T, et al. Expert Consensus Recommendations for the Suspicion and Diagnosis of Transthyretin Cardiac Amyloidosis. Circ Heart Fail 2019;12:e006075.

23. González-López E, López-Sainz Á, Garcia-Pavia P. Diagnosis and Treatment of Transthyretin Cardiac Amyloidosis. Progress and Hope. Rev Esp Cardiol (Engl Ed) 2017;70:991-1004.

24. Dungu J, Sattianayagam PT, Whelan CJ, et al. The electrocardiographic features associated with cardiac 
amyloidosis of variant transthyretin isoleucine 122 type in Afro-Caribbean patients. Am Heart J 2012;164:72-9.

25. González-López E, Gagliardi C, Dominguez F, et al. Clinical characteristics of wild-type transthyretin cardiac amyloidosis: disproving myths. Eur Heart J 2017;38:1895-904.

26. Koyama J, Falk RH. Prognostic significance of strain doppler imaging in light-chain amyloidosis. JACC Cardiovasc Imaging 2010;3:333-42.

27. Rapezzi C, Merlini G, Quarta CC, et al. Systemic cardiac amyloidoses: disease profiles and clinical courses of the 3 main types. Circulation 2009;120:1203-12.

28. Pagourelias ED, Mirea O, Duchenne J, et al. Echo parameters for differential diagnosis in cardiac amyloidosis: a head-to-head comparison of deformation and nondeformation parameters. Circ Cardiovasc Imaging 2017;10:e05588.

29. Pellikka PA, Holmes DR Jr, Edwards WD, et al. Endomyocardial biopsy in 30 patients with primary amyloidosis and suspected cardiac involvement. Arch Intern Med 1988;148:662-6.

30. Quarta CC, Gonzalez-Lopez E, Gilbertson JA, et al. Diagnostic sensitivity of abdominal fat aspiration in cardiac amyloidosis. Eur Heart J 2017;38:1905-8.

31. Ruberg FL, Maurer MS, Judge DP, et al. Prospective evaluation of the morbidity and mortality of wild-type and V122I mutant transthyretin amyloid cardiomyopathy: the Transthyretin Amyloidosis Cardiac Study (TRACS). Am Heart J 2012;164:222-228.e1.

32. Maurer MS, Hanna M, Grogan M, et al. Genotype and phenotype of transthyretin cardiac amyloidosis: THAOS (Transthyretin Amyloid Outcome Survey). J Am Coll Cardiol 2016;68:161-72.

33. Sekijima Y. Transthyretin (ATTR) amyloidosis: clinical spectrum, molecular pathogenesis and diseasemodifying treatments. J Neurol Neurosurg Psychiatry 2015;86:1036-43.

34. Grogan M, Scott CG, Kyle RA, et al. Natural history of wild-type transthyretin cardiac amyloidosis and risk stratification using a novel staging system. J Am Coll Cardiol 2016;68:1014-20.

35. Lobato L. Portuguese-type amyloidosis (transthyretin amyloidosis, ATTR V30M). J Nephrol 2003;16:438-42.

36. Bhuiyan T, Helmke S, Patel AR, et al. Pressure-volume relationships in patients with transthyretin (ATTR) cardiac amyloidosis secondary to V122I mutations and wildtype transthyretin: Transthyretin cardiac amyloid study (TRACS). Circ Heart Fail 2011;4:121-8.

37. Gertz MA, Falk RH, Skinner M, et al. Worsening of congestive heart failure in amyloid heart disease treated by calcium channel-blocking agents. Am J Cardiol 1985;55:1645.

38. Pollak A, Falk RH. Left ventricular systolic dysfunction precipitated by verapamil in cardiac amyloidosis. Chest 1993;104:618-20.

39. Rubinow A, Skinner M, Cohen AS. Digoxin sensitivity in amyloid cardiomyopathy. Circulation 1981;63:1285-8.

40. Ballantyne B, Manian U, Sheyin O, et al. Stroke risk and atrial mechanical dysfunction in cardiac amyloidosis. ESC Heart Fail 2020;7:705-7.

41. Feng D, Syed IS, Martinez M, et al. Intracardiac thrombosis and anticoagulation therapy in cardiac amyloidosis. Circulation 2009;119:2490.

42. Alkindi S, Almasoud A, Younes A, et al. Increased risk of heart block in patients with cardiac amyloidosis on amiodarone. J Card Fail 2015;21:S125.

43. Kristen AV, Dengler TJ, Hegenbart U, et al. Prophylactic implantation of cardioverter-defibrillator in patients with severe cardiac amyloidosis and high risk for sudden cardiac death. Heart Rhythm 2008;5:235-40.

44. Berk, JL, Suhr, OB, Obici K, et al. Repurposing diflunisal for familial amyloid polyneuropathy: a randomized clinical trial. JAMA 2013;310:2658-67.

45. Lin G, Dispenzieri A, Kyle R, et al. Implantable cardioverter defibrillators in patients with cardiac amyloidosis. J Cardiovasc Electrophysiol 2013;24:793-8.

46. Bulawa CE, Connelly S, Devit M, et al. Tafamidis, a potent and selective transthyretin kinetic stabilizer that inhibits the amyloid cascade. Proc Natl Acad Sci USA 2012;109:9629-34.

47. Coelho T, Maia LF, da Silva AM, et al. Long-term effects of tafamidis for the treatment of transthyretin familial amyloid polyneuropathy. J Neurol 2013;260:2802-14.

48. Maurer MS, Grogan DR, Judge DP, et al. Tafamidis in transthyretin amyloid cardiomyopathy: effects on transthyretin stabilization and clinical outcomes. Circ Heart Fail 2015;8:519-26.

49. Pfizer Inc. Summary of product characteristics. European Medicines Agency, 2011. Available online: https://www. ema.europa.eu/en/documents/product-information/ vyndaqel-epar-product-information_en.pdf (accessed 6 Apr 2020).

50. Maurer MS, Schwartz JH, Gundapaneni B, et al. Tafamidis treatment for patients with transthyretin amyloid cardiomyopathy. N Engl J Med 2018;379:1007-16.

51. Keohane D, Schwartz J, Gundapaneni B, et al. Tafamidis delays disease progression in patients with early stage 
transthyretin familial amyloid polyneuropathy: additional supportive analyses from the pivotal trial. Amyloid 2017;24:30-6

52. Klamerus KJ, Watsky E, Moller R, et al. The effect of tafamidis on the QTc interval in healthy subjects. Br J Clin Pharmacol 2015;79:918-25.

53. Sekijima Y, Dendle MA, Kelly JW. Orally administered diflunisal stabilizes transthyretin against dissociation required for amyloidogenesis. Amyloid 2006;13:236-49.

54. Castaño A, Helmke S, Alvarez J, et al. Diflunisal for ATTR cardiac amyloidosis. Congest Heart Fail 2012;18:315-9.

55. Rosenblum H, Castano A, Alvarez J, et al. TTR (Transthyretin) Stabilizers Are Associated With Improved Survival in Patients With TTR Cardiac Amyloidosis. Circ Heart Fail 2018;11:e004769.

56. Fox JC, Heitner S, Falk R, et al. AG10 Consistently stabilizes transthyretin to a high level in both wild type and mutant amyloid cardiomyopathy: responder analyses from a phase 2 clinical trial. J Am Coll Cardiol 2019;73:660.

57. Judge DP, Heitner SB, Falk RH, et al. Transthyretin stabilization by AG10 in symptomatic transthyretin amyloid cardiomyopathy. J Am Coll Cardiol 2019;74:285-95.

58. Sant'Anna R, Gallego P, Robinson LZ, et al. Repositioning tolcapone as a potent inhibitor of transthyretin amyloidogenesis and associated cellular toxicity. Nat Commun 2016;7:10787.

59. Ferreira N, Cardoso I, Domingues MR, et al. Binding of epigallocatechin-3-gallate to transthyretin modulates its amyloidogenicity. FEBS Lett 2009;583:3569-76.

60. Ferreira N, Saraiva MJ, Almeida MR. Natural polyphenols inhibit different steps of the process of transthyretin (TTR) amyloid fibril formation. FEBS Lett 2011;585:2424-30.

61. Ferreira N, Saraiva MJ, Almeida MR. Epigallocatechin-3gallate as a potential therapeutic drug for TTR-related amyloidosis: "in vivo" evidence from FAP mice models. PLoS One 2012;7:e29933.

62. Ehrnhoefer DE, Bieschke J, Boeddrich A, et al. EGCG redirects amyloidogenic polypeptides into unstructured, offpathway oligomers. Nat Struct Mol Biol 2008;15:558-66.

63. Miyata M, Sato T, Kugimiya M, et al. The crystal structure of the green tea polyphenol (-)-epigallocatechin gallate-transthyretin complex reveals a novel binding site distinct from the thyroxine binding site. Biochemistry 2010;49:6104-14.

64. Maiti TK, Ghosh KS, Dasgupta S. Interaction of (-)epigallocatechin-3-gallate with human serum albumin: fluorescence, fourier transform infrared, circular dichroism, and docking studies. Proteins 2006;64:355-62.
65. Kristen AV, Lehrke S, Buss S, et al. Green tea halts progression of cardiac transthyretin amyloidosis: an observational report. Clin Res Cardiol 2012;101:805-13.

66. aus dem Siepen F, Buss SJ, Andre F, et al. Extracellular remodeling in patients with wild-type amyloidosis consuming epigallocatechin-3-gallate: preliminary results of T1 mapping by cardiac magnetic resonance imaging in a small single center study. Clin Res Cardiol 2015;104:640-7.

67. Dias N, Stein CA. Antisense oligonucleotides: basic concepts and mechanisms. Mol Cancer Ther 2002;1:347-55.

68. Bennett CF. Therapeutic antisense oligonucleotides are coming of age. Annu Rev Med 2019;70:307-21.

69. Lasser K, Hoch JS, Mickle K, et al. Inotersen and patisiran for hereditary transthyretin amyloidosis: effectiveness and value. Final evidence report. Institute for Clinical and Economic Review. October 4, 2018. (accessed April 2nd 2020). Available at: https://icer-review.org/wp-content/ uploads/2018/02/ICER_Amyloidosis_Final_Evidence_ Report_100418.pdf

70. Meister G. Argonaute proteins: functional insights and emerging roles. Nat Rev Genet 2013;14:447-59.

71. Suhr OB, Coelho T, Buades J, et al. Efficacy and safety of patisiran for familial amyloidotic polyneuropathy: a phase II multi-dose study. Orphanet J Rare Dis 2015;10:109.

72. Coelho T, Adams D, Silva A, et al. Safety and efficacy of RNAi therapy for transthyretin amyloidosis. N Engl J Med 2013;369:819-29.

73. Butler JS, Chan A, Costelha S, et al. Preclinical evaluation of RNAi as a treatment for transthyretin-mediated amyloidosis. Amyloid 2016;23:109-18.

74. Alnylam Pharmaceuticals Inc. Summary of Product Characteristics. European Medicines Agency, 2018. (accessed 6 Apr 2020). Available online: https://www.ema. europa.eu/en/documents/product-information/onpattroepar-product-information_en.pdf

75. Adams D, Gonzalez-Duarte A, O'Riordan WD, et al. Patisiran, an RNAi therapeutic, for hereditary transthyretin amyloidosis. N Engl J Med 2018;379:11-21.

76. Solomon SD, Adams D, Kristen A et al. Effects of patisiran, an RNA interference therapeutic, on cardiac parameters in patients with hereditary transthyretinmediated amyloidosis. Circulation 2019;139:431-43.

77. Judge DP, Kristen AV, Grogan M, et al. Phase 3 Multicenter Study of Revusiran in Patients with Hereditary Transthyretin-Mediated (hATTR) Amyloidosis with Cardiomyopathy (ENDEAVOUR). Cardiovasc Drugs Ther 2020;34:357-70. Erratum in: Cardiovasc Drugs Ther. 2020 Jun 16. doi: 10.1007/s10557-020-07023-8. Online 
ahead of print.

78. Ackermann EJ, Guo S, Benson MD, et al. Suppressing transthyretin production in mice, monkeys and humans using 2nd-generation antisense oligonucleotides. Amyloid 2016;23:148-57.

79. Benson MD, Waddington-Cruz M, Berk JL, et al. Inotersen treatment for patients with hereditaty transthyretin amyloidosis. N Engl J Med 2018;379:22-31.

80. Benson MD, Dasgupta NR, Rissing SM, et al. Safety and efficacy of a TTR specific antisense oligonucleotide in patients with transthyretin amyloid cardiomyopathy. Amyloid 2017;24:219-25.

81. Dasgupta NR, Falk RH, Drachman BM, et al. Safety of inotersen treatment in patients with transthyretin amyloid cardiomyopathy. J Am Coll Cardiol 2019;73:909.

82. Akcea Therapeutics Ltd. Summary of Product Characteristics. European Medicines Agency, 2018. (accessed 6 Apr 2020). Available online: https://www.ema. europa.eu/en/documents/product-information/tegsediepar-product-information_en.pdf

83. Ward JE, Ren R, Toraldo G, et al. Doxycycline reduces fibril formation in a transgenic mouse model of AL amyloidosis. Blood 2011;118:6610-7.

84. Cardoso I, Martins D, Ribeiro T, et al. Synergy of combined doxycycline/TUDCA treatment in lowering transthyretin deposition and associated biomarkers: studies in FAP mouse models. J Transl Med 2010;8:74.

85. Karlstedt E, Jimenez-Zepeda V, Howlett JG, et al. Clinical experience with the use of doxycycline and ursodeoxycholic acid for the treatment of transthyretin cardiac amyloidosis. J Card Fail 2019; 25: 147-53.

86. Obici L, Cortese A, Lozza A, et al. Doxycycline plus tauroursodeoxycholic acid for transthyretin amyloidosis: a phase II study. Amyloid 2012;19:34-6.

87. Galant NJ, Bugyei-Twum A, Rakhit R, et al. Substoichiometric inhibition of transthyretin misfolding

Cite this article as: Cruz Rodriguez JB, Tallaj JA. Narrative review of pharmacotherapy for transthyretin cardiac amyloid. Ann Transl Med 2021;9(6):519. doi: 10.21037/atm-20-4636 by immune-targeting sparsely populated misfolding intermediates: a potential diagnostic and therapeutic for TTR amyloidoses. Sci Rep 2016;6:25080

88. Phay M, Blinder V, Macy S, et al. Transthyretin aggregatespecific antibodies recognize cryptic epitopes on patientderived amyloid fibrils. Rejuvenation Res 2014;17:97-104

89. Ando Y, Ueda M. Antibody therapy for transthyretinrelated hereditary amyloid polyneuropathy: another therapeutic option. Amyloid 2017;24:113-4.

90. Higaki JN, Chakrabartty A, Galant NJ, et al. Novel conformation-specific monoclonal antibodies against amyloidogenic forms of transthyretin. Amyloid 2016;23:86-97.

91. Bodin K, Ellmerich S, Kahan MC, et al. Antibodies to human serum amyloid $\mathrm{P}$ component eliminate visceral amyloid deposits. Nature 2010;468:93-7.

92. Pepys MB, Herbert J, Hutchinson WL, et al. Targeted pharmacological depletion of serum amyloid $\mathrm{P}$ component for treatment of human amyloidosis. Nature 2002;417:254-9.

93. Richards DB, Cookson LM, Barton SV, et al. Repeat doses of antibody to serum amyloid $\mathrm{P}$ component clear amyloid deposits in patients with systemic amyloidosis. Sci Transl Med 2018;10:eaan3128.

94. Dubrey SW, Davidoff R, Skinner M, et al. Progression of ventricular wall thickening after liver transplantation for familial amyloidosis. Transplantation 1997;64:74-80.

95. Ericzon BG, Wilczek HE, Larsson M, et al. Liver transplantation for hereditary transthyretin amyloidosis: after 20 years still the best therapeutic alternative? Transplantation 2015;99:1847-54.

96. Careddu L, Zanfi C, Pantaleo A, et al. Combined heartliver transplantation: a single-center experience. Transpl Int 2015;28:828-34.

97. Sousa M, Monohan G, Rajagopalan N, et al. Heart transplantation in cardiac amyloidosis. Heart Fail Rev 2017;22;317-27. 